STAIF Conference on Innovative Transportation Systems for Exploration of the Solar System and Beyond, Albuquerque NM, Feb. 11-15, 2001

\title{
Interstellar Flight by Particle Beam
}

\author{
Geoffrey A. Landis \\ NASA John Glenn Research Center, mailstop 302-1, 21000 Brookpark Road, Cleveland, OH 44135 \\ (216) 433-2238 e-mail: geoffrey.landis@grc.nasa.gov
}

\begin{abstract}
Two difficulties with the use of laser-propelled lightsails for interstellar propulsion are the extremely low energy efficiency, and the extremely large lenses required. Both the energy efficiency and the required lens size may be greatly improved by use of a particle beam, rather than a light beam. The particle beam is reflected by a magnetic field on the spacecraft, for example, by a magnetic sail or a mini-magnetosphere inflated by a plasma current. This results in a net force on the sail with no expenditure of propellant, allowing extremely high delta-V missions, such as an interstellar probe, to be accomplished.
\end{abstract}

\section{INTRODUCTION}

With the discovery of planets around nearby stars, the idea of sending an interstellar probe to investigate planetary systems of other stars has become a highly visible project at NASA. However, the extremely high delta-V required for an interstellar probe makes conventional rocket propulsion problematical. It would be desirable to send an interstellar probe at a velocity high enough to return data within the lifetime of the scientists who launched the probe, and this cannot be accomplished with any existing rocket technology.

A proposed solution is to use beamed-energy propulsion, where the power source remains stationary, and a beam transfers the energy to the spacecraft. By the stratagem of not carrying the power source onboard the vehicle, the exponentially-increasing mass ratio of a rocket is avoided. One such beamed-energy propulsion system is the laserpushed lightsail (Forward, 1984).

A difficulty with the use of laser-propelled lightsails for interstellar propulsion is the extremely low energy efficiency, 6.7 N/GW. The inefficient use of energy means that extremely large power sources are required, which increases the cost (Andrews, 1993; Landis, 1997). Typical proposals, for example, require lasers with power in the range of 25-50 gigawatts (Landis, 1997, 1999) to 7.2 terawatts (Forward, 1984).

A second problem with laser-pushed sails is the requirement for extremely large lenses to avoid diffraction of the light over interstellar distances, typically in the hundreds of kilometers in diameter (Landis, 1997, 1999) to as much as thousands of kilometers for rendezvous mission architectures (Forward, 1984). These considerations mean that laser-pushed lightsails are impractical in the reasonably foreseeable future.

Both the energy efficiency and the required lens size may be greatly improved by use of a particle beam, rather than a light beam, to push the sail. A particle-beam pushed sail was proposed by Landis (1989), and an analysis of the cost of such a proposal was done by Andrews (1993). In this propulsion system, a charged particle beam is accelerated, focused, and directed at the target; the charge is then neutralized to avoid beam expansion due to electrostatic repulsion. The particles are then be re-ionized at the target and reflected by a magnetic sail, resulting in a net momentum transfer to the sail equal to twice the momentum of the beam. This magnetic sail was originally proposed to be in the form of a large superconducting loop with a diameter of many tens of kilometers, or "magsail"; (Andrews and Zubrin, 1988). The recent development of the mini-magnetosphere ("M2P2") magnetic sail, proposed by Winglee et al. $(2000,2001)$, makes the required size of the physical structure of the sail much smaller, and hence makes the particle-beam pushed propulsion system more practical.

This is a preprint or reprint of a paper intended for presentation at a conference. Because changes may be made before formal publication, this is made available with the understanding that it will not be cited or reproduced without the permission of the author. 


\section{PARTICLE-BEAM INTERSTELLAR SAIL}

A particle-beam pushed sail has many advantages as a propulsion system for interstellar flight:

1. Light weight. Since the sail reflecting the beam is not a physical object, it can be made extremely light.

2. Large target. The actual reflecting area of a mini-magnetosphere sail is much larger than the magnetic loop itself. Therefore, the sail can be quite large. This makes the aiming, beam stabilization, and beam divergence problem much lower.

3. High acceleration. The limit on the acceleration of a lightsail is set by the temperature limit of the sail material (Landis, 1997, 2000). Since the active part of a mini-magnetosphere magnetic sail is, in fact, a magnetic field, there is not a significant thermal limit. The field is sustained by a plasma, which likewise is not subject to melting. Only the physical magnet itself is thermally limited, and this magnet is extremely small compared to the sail area. By increasing the acceleration, cruise velocity can be achieved in a shorter distance, again decreasing the requirements for beam divergence and the aiming and stabilization difficulty.

4. Higher momentum/energy ratio. A particle beam has a much more momentum per unit energy than a photon (e.g., laser) beam, and hence transfers force to a sail with better energy efficiency. The (relativistically correct) relation between momentum $(p)$ and energy $(E)$ for both particle beams and photon beams is:

$$
\mathrm{p}^{2}=\mathrm{E}^{2} / \mathrm{c}^{2}+\mathrm{m}_{\mathrm{o}} \mathrm{E}
$$

(note that energy here refers only to the applied kinetic energy of the beam, not including the rest-energy $\mathrm{m}_{0} \mathrm{c}^{2}$ of the particles). For a photon beam, the rest mass $m_{o}$ is zero. It is clear that the ratio of momentum to energy increases directly as the rest mass of the particles composing the beam increases. Since the ratio of momentum to energy equals force produced on the sail per unit of beam power, a particle-beam pushed sail has a higher energy efficiency.

5. Beam energy efficiency. Lasers have relatively low efficiency of conversion of electrical energy, typically less than $25 \%$ for lasers of the beam quality required. $50 \%$ conversion efficiency is reasonable to assume for future lasers. The energy efficiency of a particle beam can be made extremely high. In principle efficiencies of well over $90 \%$ should be achievable. This reduces the input power requirements, and also reduces the requirement for heat radiators to reject the waste heat.

6. Low beam divergence. The large size of the laser-pushed sail and lens is required because a laser beam diameter, over the acceleration distances required for interstellar propulsion, is limited by diffraction of the laser beam.

For a particle beam, beam spread due to diffraction is not a problem, and hence the large diameter of the lens used in laser sail proposals is no longer needed. The particles would have to be charged to be accelerated and focused, and then neutralized to avoid beam expansion due to electrostatic repulsion. The particles could then be re-ionized and reflected by a magnetic sail.

One potential difficulty is beam spread due to random thermal velocity. Thermal beam spread decreases proportionately to the square root of the atomic mass:

$$
\mathrm{v}=\left(\mathrm{kT} / \mathrm{m}_{\mathrm{o}}\right)^{1 / 2}
$$

where $\mathrm{k}$ is the Boltzmann constant and $\mathrm{T}$ is the beam temperature.

Using mercury (atomic mass 80 ) as an example, the lateral velocity due to thermal motion is $10 \sqrt{T} \mathrm{~m} / \mathrm{sec}$, where $T$ is the temperature in Kelvin. Existing ion accelerators have typical beam temperatures of hundreds to thousands of degrees, but this can in principal be reduced by any of several known techniques. A reasonable limit to the minimum temperature that can be theoretically achieved for a beam is about $45 \mathrm{~K}$ might be achieved, typical of the temperature of material in thermal equilibrium with starlight. This would lead to a thermal beam divergence of about 2 million kilometers per light year.

This could be reduced if the beam particles condense to larger particles after acceleration. To reduce the beam spread by a factor of a thousand, the number of mercury atoms per condensed droplet needs to be at least a million. This is an extremely small droplet $\left(10^{-16}\right.$ grams) by macroscopic terms, and it is not unreasonable to believe that such condensation might take place in the beam. As the droplet size increases, this propulsion concept approaches that of momentum transfer by use of pellet streams, considered for interstellar propulsion by Singer (1980). 


\section{USE OF MINI-MAGNETOSPHERE}

By shrinking the physical structure of a magnetic sail, the invention of the mini-magnetosphere plasma propulsion, or "M2P2" (Winglee et al. 2000, 2001), has brought the idea of a particle-beam pushed sail closer to reality. The particle beam is reflected by a magnetic field. In the mini-magnetosphere, the magnetic field is inflated to large areas by the injection of a plasma, and hence large magnetic field areas are possible with only a small physical structure.

A difficulty of the mini-magnetosphere is that the plasma itself slowly leaks away, and must be replenished from an on-board source. Unlike a solar sail, a mini-magnetosphere requires propellant, and if on-board propellant is used at the high delta- $V$ required for an interstellar probe, the mass ratio becomes prohibitive.

For the interstellar propulsion system, I propose that the replenishment of the plasma be accomplished directly from the particle beam. This is relatively straightforward. The particle beam must be ionized to be reflected from the sail, but if the ionization is not perfect, the beam will contain a number of neutrals. These neutrals will penetrate the magnetic field. Once inside the magnetic field, they will collide with the existing ions in the plasma current, and since the beam energy is much greater than the ionization energy of the atoms, the collisions will result in ionization. Thus, the mini-magnetosphere will self-inflate with the particle beam, resulting in no requirement for on-board propellant except, possibly, for a small amount for initial inflation of the magnetic field.

An additional advantage of using a magnetic sail for interstellar propulsion is that the same sail can be used for both propulsion and also to slow the probe at the destination by drag against the interstellar medium and the stellar wind of the target star. A braking system considerably improves the data return of an interstellar probe, since a fly-by probe moving at typical interstellar speeds of $10 \%$ of the speed of light will spend only an hour or so in the close neighborhood of the target. Use of magnetic sails for braking is discussed by Andrews and Zubrin (1988).

A secondary advantage of using a mini-magnetosphere plasma is that the plasma will serve as a shield against impact by interstellar dust. This application has been briefly discussed in (Landis, 1994).

\section{CONCLUSIONS}

The development of mini-magnetosphere plasma propulsion (M2P2) makes the concept of interstellar propulsion using a particle-beam pushed sail more attractive. A beam-pushed sail has the advantage of allowing the power source to remain fixed, and thus allows high delta- $V$ without exponential increase in mass ratio, and the particlebeam has several advantages over a laser beam to push a sail, including higher energy efficiency, higher acceleration, and a lighter sail. By using the particle beam itself to supply the ions to inflate the magnetic field, the requirement for a propellant source is removed.

Of course, the utility of a particle-beam pushed propulsion system is not limited to interstellar missions, but could be applied to a wide number of other applications. Some of the possible nearer-term applications include missions to the outer planets, the Kuiper belt, Oort cloud, and the solar heliopause. The propulsion system could also be used for short trip-time missions within the inner solar system.

\section{ACKNOWLEDGEMENTS}

This work was done while the author was the senior scientist at the Ohio Aerospace Institute (OAI), and was supported in part by a fellowship from the NASA Institute for Advanced Concepts (NIAC). 


\section{REFERENCES}

Andrews, D. G., and Zubrin, R. M., "Magnetic Sails and Interstellar Travel," IAF Paper IAF-88-5533, Bangalore India, October 1988.

Andrews, D. G., "Cost Considerations for Interstellar Missions," paper IAA-93-706 (1993); also presented at Conference on Practical Robotic Interstellar Flight, New York University, August 29-Sept. 1, 1994.

Forward, R. L., "Roundtrip Interstellar Travel Using Laser-Pushed Lightsails," J. Spacecraft and Rockets, Vol. 21, Mar-Apr. 1984, pp. 187-195.

Landis, G. A., "Optics and Materials Considerations for a Laser-propelled Lightsail," Paper IAA-89-664, 40th IAF Congress, Torremolinos Spain, Oct. 7-13, 1989.

Landis, G. A., "High Performance Laser-Pushed Lightsails: Materials Considerations," DOE Workshop on Beamed Power, PNLSA-19599, p. 28, and supplement, pp. TNP1 1-3 (1991).

Landis, G. A., "Erosion Shields for Interstellar Dust," Planetary Society Conference on Practical Robotic Interstellar Flight, NY University, Aug 29-Sept. 1, 1994.

Landis, G. A., "Small Laser-Pushed Lightsail Interstellar Probe," Journal of the British Interplanetary Society, Vol. 50, No. 4, 149-154 (1997); Paper IAA-95-4.1.1.02, presented at the 46th International Astronautics Federation Congress, Oslo Norway, 2-6 Oct. 1995.

Landis, G. A., "Beamed Energy Propulsion for Practical Interstellar Flight," Journal of the British Interplanetary Society, Vol. 52, 420-423 (1999).

Landis, G. A., Dielectric Films for Solar- and Laser-pushed Lightsails," AIP Conference Proceedings Volume 504, presented at Space Technology and Applications International Forum (STAIF-2000), Jan. 30 - Feb. 3, 2000, Albuquerque NM.

Singer, C. E., "Interstellar Propulsion Using a Pellet Stream for Momentum Transfer, " J. British Interplanetary Soc., Vol. 33, Mar. 1980, pp. 107-115.

Winglee, R. M., Ziemba, T., Slough, J., Euripides, P., and Gallagher, D., "Laboratory Testing of the Mini-Magnetospheric Plasma Propulsion (M2P2) Prototype," presented at Space Technology and Applications International Forum (STAIF-2001), Feb. 11-15 2001, Albuquerque NM.

Winglee, R. M., Slough, J., Ziemba, T., and Goodson, A., "Mini-magnetospheric Plasma Propulsion: High speed propulsion sailing the solar wind," Space Technology and Applications International Forum-2000, edited by M. S. El-Genk, American Institute of Physics CP504, 1-56396-9, p. 962, Feb. 2000. 